\title{
Management of crisis exercises in Virtual Reality
}

\author{
Alexis Evain* \\ IMT Mines Albi
}

Aurélie Congès †

IMT Mines Albi
Frederick Benaben $\ddagger$

IMT Mines Albi

\begin{abstract}
When we consider the question of crisis management, training and practising exercises are regularly requested to be ready for any possible crisis. Unfortunately, these exercises come with many disadvantages, especially the cost, the complexity of implementation, the lack of replicability, and agility. The project aims to create customizable Virtual Reality simulations in existing environments. In this article, we detail our approach dedicated to using VR for a fire in a University Research Lab involving a game master managing the scenario, five people, interacting and acting conjunctly within this environment, and several coordinators the rescue process.
\end{abstract}

Keywords: Virtual Reality, training, teaching, collaboration.

Index Terms: K.6.1 [Management of Computing and Information Systems]: Project and People Management—Life Cycle; K.7.m [The Computing Profession]: Miscellaneous-Ethics

\section{INTRODUCTION}

In crisis management, training and preparation are essential: "exercises are a fundamental part of building the necessary capacity and required capabilities to effectively deal with information during and after disasters" [19]. Training exercises are often mandatory in critical sites, which must set up exercises several times a year, depending on the legislation and the site, to be ready to face any kind of crisis. In this article, "critical sites" refers to sites in which the users' life can be endangered by dysfunctions or incidents. However, despite the technological advances, crisis management training continues to be "paper-based, collective group dynamic exercises" [2], which presents limitations.

Paper-based or classroom exercises even for table exercises have little retention potential: as presented in [25], the average retention rate of information following teaching is only $5 \%$ when students just listen, and $10 \%$ when they read. On the contrary, the retention rate of information is much higher if students are active rather than passive: $75 \%$ of information is retained when trainees practise by doing. However, simulation-based exercises in real size also have drawbacks. As explained in [4], exercises are expensive, they require a lot of time and resources, and they lack realism and flexibility.

\footnotetext{
* e-mail: $\underline{\text { alexis.evain@mines-albir.fr }}$

$\dagger$ e-mail: aurelie.conges@mines-albi.fr

† e-mail: frederick.benaben@mines-albi.fr
}

Mobilizing actors for exercises is difficult, it is hard to predict their implications and how impacted they will be. Immobilizing a site for the duration of the exercise is costly, and thus it cannot be done often enough to cover any type of crisis that might occur. As explained in [1], "traditional training simulated-based systems are expensive and offer little flexibility", and "real training in the field of emergency management is very expensive and complicated regarding the harmonization of procedures between agencies". Consequently, the frequency and coverage of the crisis scenario are not high enough. Besides, the realism of the scenario is hard to get, as, for instance, it is not possible to burn a building every time we want to practice putting out a fire.

To overpass those limitations, virtual exercises are already used in the aviation, military, or medical fields [15]. Those virtual exercises can be performed through keyboard and mouse, controllers, or immersive Virtual Reality, and they allow students to gain skills through a safe and guided practice [17]. Based on those observations, this research project proposes to use Virtual Reality to build a training platform dedicated to crisis management.

This article is structured as follows: first, an overview of the use of Virtual Reality as a crisis management training tool. Then, the presentation of the project Virtual Reality training platform and its architecture, as well as a use case showing how it can be used and an evaluation of the benefits. Finally, the presentation of the project's next steps and perspectives.

\section{Context}

According to the Oxford dictionary, learning is "the acquisition of knowledge or skills through study, experience, or being taught" [26]. In [9], Gerard proposes a decomposition of knowledge in three activities: know how to reproduce (being able to reproduce or repeat a message, a gesture, an action, without transformation and out of any context), know how to do (being able to perform a learned action in a new and appropriate context), and know-how to be (being able to apprehend situations and other actors and adapt one's actions and reactions).

We propose to add a first and more basic step to those activities: know (mastering the theory before trying to repeat a message or an action). Before he can operate, a surgeon needs to master several skills. First, he needs to gain knowledge of the human body and the organs he will have to operate (know). Then, he needs to master the necessary actions to operate: how to use a scalpel, how to sew skin, etc. (know how to reproduce). He also needs to know the order in which he has to perform the different tasks during the operation: the process he must follow (know how to do). And finally, the surgeon needs to know how to work with the people present in the operating room with him: nurses, anaesthetists, other doctors, etc. That is the collaborative aspect (know how to be). Those four steps (knowledge, action, process, and collaboration) will constitute our decomposition of learning levels, that we will use in this article. 


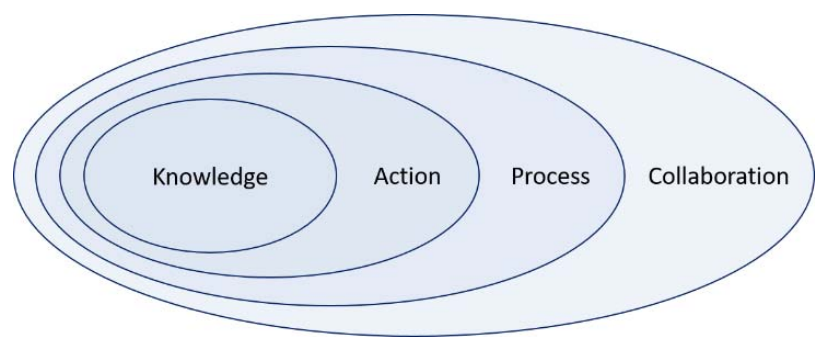

Figure 1: Our decomposition of learning levels

To master those steps, class-room exercises are not enough, and trainees need to practice in a realistic situation. Virtual Reality presents several characteristics that make it a great tool for learning and practising within those four steps and offer solutions to the drawbacks of classical exercises. In this section, we are going to explain why. Then, we will see how those characteristics can be used to improve training in crisis management.

\subsection{Virtual Reality: a great learning tool}

Virtual Reality can help trainees gain theoretical knowledge thanks to its ability to faithfully represent things in $3 \mathrm{D}$ on the one hand, and the other hand to its ability to "extend the user's capability to feel data that would normally be beyond the range of their sensed or experiences" [20]. As stated in [18], if you want to teach biology, there is no point in building a virtual classroom if you can build a realistic and interactive human cell. Virtual Reality allows scalability, which gives "the users the ability to 'change' their physical size so that they can navigate and interact in macro and micro worlds" [20]. This possibility can also be used in data visualization or analysis, which can also help to gain theoretical knowledge. For instance, the AR bone puzzle [24] uses augmented reality to help medical students to learn human bone anatomy. The system uses interactions based on intuitive gestures (grasping, moving, releasing) and the 3D aspects offered by augmented reality to help students to learn. Even if that particular game uses augmented reality, it is based on features that also exist in Virtual Reality. This could also bring more possibilities to help students visualize and manipulate objects to better understand how they are made or how they work. The VR Anatomy System [7] is another example of an educational tool in Virtual Reality. This system aims at teaching medical students the anatomy and functioning of a human heart through a realistic and interactive 3D model and was positively evaluated by medical professionals.

The practical knowledge (action) is probably the one that benefits the most from the advantages of Virtual Reality. According to [8], "Virtual Reality is a three-dimensional user interface in which the user can perform actions and experience their consequences". Immersion and interaction with the environment are key features of Virtual Reality that allows trainees to practice specific gestures and actions. As stated in the introduction, the retention potential for students is much higher when they can practise by doing, and Virtual Reality offers that possibility: "interactions are central to all Virtual Reality training simulations. They are what define the user experience and allow the simulation to be an effective training tool" [21]. This possibility to interact with the environment, along with the feeling of immersion induced by Virtual Reality, allow a bigger involvement of the trainees, which are then more committed to the exercise and abler to learn, as shown in [20]. Practising gestures in Virtual Reality can for instance be used in sports: ballet dancers can

\footnotetext{
${ }^{1}$ https://immersivefactory.com/
}

practice movements and receive feedbacks [16]. In the medical field, [11] proposes a system to train surgeons for knee arthroscopic surgery. This system provides realistic visual and haptic feedback that allows students to train efficiently for this surgery, and thus limit the potential damages done to the patient.

One of the weaknesses of classical exercises is that they are not frequent enough for trainees to be prepared for any kind of crisis. Not all the conditions or possibilities can be recreated or studied in a classroom exercise, and they lack realism. For instance, "the acquisition of clinical skills by medical trainees can be haphazard, relying on the chance presentation of patients with specific diseases or surgical conditions" [21]. Virtual Reality allows us to create exercises representing a wide range of conditions, with "customized learning, challenging environment, and multi-sensory effects" [12]. The immersion and involvement of users make the exercises more realistic and impactful. Besides, it is possible to create a consistent scenario that can be repeated as often as needed [6], [13] with no further costs. If it is not possible to burn down a building to practice fire management, "for training and education purposes, Virtual Reality allows trainees to practice cognitive, and sometimes physical, skills in simulation as risks to safety and material costs can be much lower in Virtual Reality than in physical training" [14]. This reduction of risks also allows learning by trial and error: "Players are encouraged to learn by trying different strategies, succeeding and failing as they would in an entertainment game" [22]. This can be used to teach processes. Immersive Factory ${ }^{1}{ }^{1}$ proposes Environments, Health, and Safety workshops in Virtual Reality where trainees must perform some tasks such as replacing a bulb in height or using a forklift truck. Mistakes and missed steps in the process cause injuries to the avatar, and the end of the training session. However, the user remains safe, and can thus learn from his mistakes and try new solutions without risks. Mimbus $C^{2}$ proposes Virtual-Indus, a tool dedicated to teaching industrial procedures such as electrical intervention or maintenance procedures. Virtual-Indus offers a realistic environment in which it is possible to implement a scenario that would be too dangerous or too expensive in real life.

Several studies have proven that Virtual Reality has great potential for learning and practising. [10] findings prove that "virtual environments fulfil their promise of providing repeated practice opportunities in disperse locations with uncommon, lifethreatening trauma cases in a safe, reproducible, flexible setting" while being "more cost-effective than many other methods". Studies involving 16 surgical residents show that those training with Virtual Reality can perform operations $29 \%$ faster than those trained traditionally [23].

In crisis management, exercises, though mandatory, present several drawbacks. In the next section, we are going to see how those drawbacks can be addressed by Virtual Reality.

\subsection{Virtual Reality for crisis management}

In [10], Heinrichs sums up the advantages of virtual worlds training:

- the context (layout, resources) can be exactly replicated,

- trainees can participate in the exercises from different locations,

- exercises can be conducted at any time without having to wait for specific conditions since multiple scenario and conditions can be recreated in the virtual environment,

\footnotetext{
${ }^{2}$ https://www.mimbus.com/portfolio/virtual-indus/
} 
- $\quad$ scenarios can be repeated as many times as needed in a safe environment, allowing trainees to learn from their mistakes,

- $\quad$ recording an exercise is possible, which enables analyses of trainees' performances.

Those advantages can be applied to crisis management, to improve the responders' preparation. In a crisis, decisions and actions have to be precise, quick, and effective in a stressful and urging context. This means that the crisis response's actors need to master skills perfectly, to be able to use them during the crisis. Thus they need to train in realistic environments "to ensure that these skills can be used in emergencies and while under stress" [19]. Virtual Reality gives the possibility to train in the digital twin of a site, under specific chosen conditions, and to repeat a crisis scenario as many times as needed to master skills and be able to rely on them in a real crisis. Because of the digital aspect of Virtual Reality, it is also possible to record the training and thus to watch it and analyse it to improve.

Crisis management is an area in which there are risks to people, civil casualties as well as crisis responders. Simulation-based training offers the advantage to practice in realistic and safe conditions, where mistakes can be made without endangering people or materials. "The use of simulators can help a lot in communicating emergency procedures to a wide range of people, by challenging them in a variety of foreseeable crises without running the risk of exposing them to real risks before they are fully aware and trained, with the further benefit of achieving, through an immersive situation, better retention of information received" [8].

When a crisis occurs, several actors are involved in the response: firemen, policemen, medical doctors, sometimes some other professionals. Each of them has its way of communicating, its professional language, and it can be difficult for them to communicate and to work together in the most effective way, which means that the collaborative aspect in the four learning steps of Figure 1 is crucial in crisis management.

Based on the advantages of Virtual Reality as a training and learning tool, we want to propose a virtual environment dedicated to the training of crisis responders. In the next section, we are going to present our proposal, and how it will improve crisis management preparation by helping trainees gain knowledge about theory, practice, processes, and collaboration.

\section{Approach}

\subsection{Introduction}

To make this training in Virtual Reality we start to create one use case defined with basics intentions. The simulation had to include collaboration and dedicated exercise according to each trade. The first use case has been developed and proposed here [4]. It allowed people to choose between the roles of doctor, firefighter, and police officer, each with their mission. The environment was a metro where the fire did not spread as it went along, and injured people had to be found.

This simulation in a fictitious location was based on the principle of declaring a process for 3 users. Each had their dedicated tasks according to their roles and from start to finish had the same

\footnotetext{
${ }^{3}$ https://unity.com/

${ }^{4} \mathrm{https} / / /$ www.unrealengine.com/

${ }^{5} \mathrm{https}$ ://godotengine.org/

${ }^{6} \mathrm{https}: / /$ www.babylonjs.com/
}

mission. They each had their tools from the beginning and therefore had only one interaction available apart from moving around.

For this use case, we use Unity ${ }^{3}{ }^{3}$ and like the previous, we made this choice by comparing several technologies. One of the most important points is to have a quick and easy Virtual Reality preview of the chosen decor. Compared to Unreal Engine $C^{4}$, Godot $C^{5}$ or Babylon $\bigodot^{6}$ it turns out that for building research prototypes Unity $₫$ is easier to access for the developers in our team. Its ergonomics and robustness will allow it to quickly go further than others and be supported by a wide variety of our machines. Working with Unreal Engine was considered but this would have resulted in too much learning when the foundations are already built with Unity. And finally, the graphical rendering achieved with Unreal Engine is compensated by the new rendering brought by the latest Unity advances. The $\mathrm{C \#}$ is a pure object-oriented modern language. $\mathrm{C \#}$ programming is often be used to create mobile and console applications and this is helping for our training who want to develop a small project on their machine.

When using Virtual Reality, several technologies are available and robust enough to be used for research. For this case, the most appropriate being HTC Vive ${ }^{7}{ }^{7}$ we have multiplied them to have a total of five people connected. The latter being wireless they were able to be free to move freely. Depending on the script we arrange the equipment in wireless or wired to obtain or better capture. We have kept the wireless for the majority of the time because in order to free ourselves from the KatVR if necessary and to be able to have someone try it out who could visit or participate in an event as a spectator, we have kept the wireless for the majority of the time. Three of them were placed on $\mathrm{KatVR}^{8}{ }^{8}$, which allowed us to optimize the demonstration space. For the moment they are only used to allow easy restriction of the playing areas. It is planned to use them more extensively.

The integration is made by the Software Development Kit: SteamVRC ${ }^{9}$. It is the common one and the most accessible to use. For its use, only footprints have been added instead of the teleport cursor. These footprints rotate according to where the gaze turns. Moreover, we have modified the teleportation to be a linear movement so that it can be understood and not disturbing by the players who could interact with it.

For multiplayer, the simulation uses the asset PUN $2^{10}$ on the asset store which has the advantage of being free and above all accessible very quickly. Their service offers voice and text chat in addition to their more classic service. A way to extend the link between players in real-time. This communication extends the possibilities of collaboration. Other technologies exist, such as the one Unity has set up with UNet, but it has been put on hold until a better service can be offered.

Now it is possible to demonstrate more advanced agility in our use case. To respond to a major crisis that evolves in real-time in an existing site.

\subsection{Scenario}

We would like to have a simulation that can be used for training on the sites that are selected. The scenario has been written with the help of a professional group composed of firefighters and a person from the security services of the site. The advantage of this result

\footnotetext{
${ }^{7} \mathrm{https}: / /$ www.vive.com/

${ }^{8} \mathrm{https}: / /$ www.kat-vr.com/

${ }^{9} \mathrm{https} / / / \mathrm{www}$. steamvr.com/

${ }^{10} \mathrm{https} / / /$ www.photonengine.com/en-us/Photon
} 
is that it can be shown to all the personnel of the chosen location. And to have the potential to apply and exploit it in different ways.

In the scenario, six people are connected in the same virtual environment. Here are the roles for users: the Game Master, two civils: an Assistant and a Technician and also three firefighters: a Chief Firefighter and two Firefighters.

Around them is a replica representing our lab building. The Game Master authorized to trigger events will launch a first fire start. This will cause an alert to the technical service player who will confirm the fire.

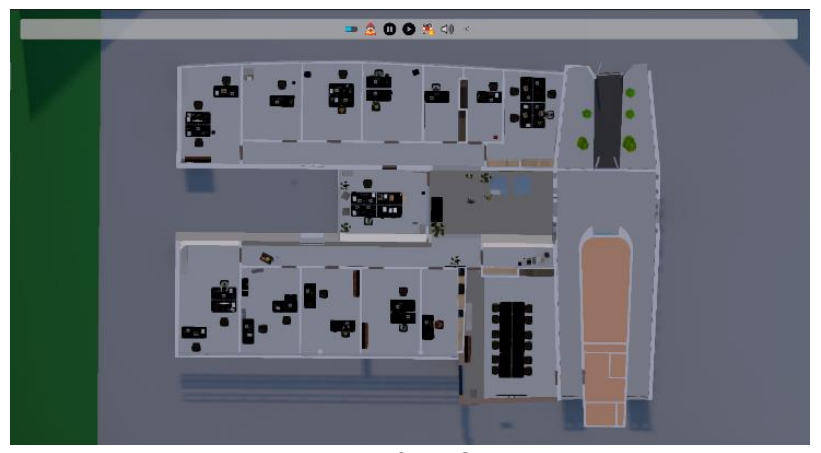

Figure 2: View of the Game Master

Once confirmed, the school alarm goes off and causes the exit of the non-player characters as well as the player from the technical department who has finished his scenario (Fig 3). The player in charge of checking that the offices are empty goes around the school. He notices one person still working because the character was wearing a headset and did not hear the alarm. When the player enters the room, he signals the alert, which makes the person leave. The player continues his way and sees a second fire with a person trapped by the fire as well as shouting behind a locked door. He goes out and warns the firefighters who have just arrived. The firefighters receive their tasks sent by the decision support platform and go to fulfil them. The smoke sensors have sent the decision support platform information about the position of the fires.

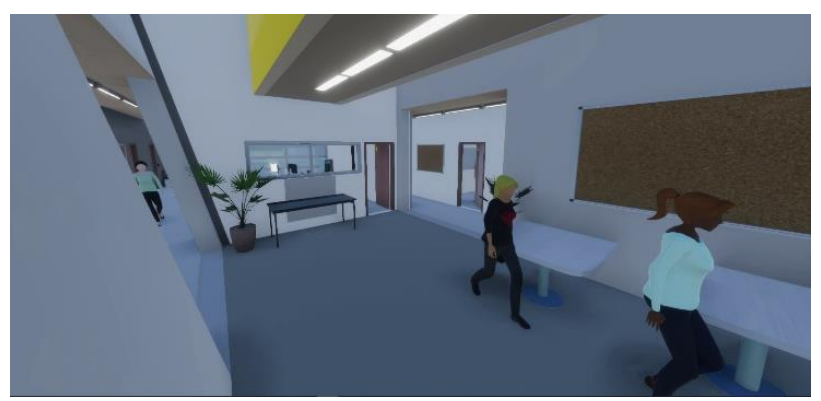

Figure 3: People exit the school

The first firefighter extinguishes the fire at the end of the corridor to free the person behind. The second firefighter has the task of extinguishing the first fire that has spread throughout the office and spread to the adjoining offices. The last firefighter head supervises the relationship between the firefighters and the civilians outside as well as with the people in charge of the establishment.

At the same time, the Game Master can add new fires to make it harder for the fire brigade.

Outside the firefighter collects new information: a gas bottle was stored in an office and screams were heard behind a door. The firefighter informs the crisis unit which modifies the response process via the decision support platform as the context is no longer the same. As this process is different, the fire brigades on-site are notified of the changes and must operate differently. One must force a door and evacuate a civilian while the other must retrieve the gas cylinder.

This fire team had come because they were already close to the school, but they did not have the necessary equipment. So, they operated while waiting for the arrival of better-equipped backup. The spread of the fire does not allow the firefighters to stay too long because the smoke becomes too present and they, unfortunately, must evacuate the premises now that there is more danger.

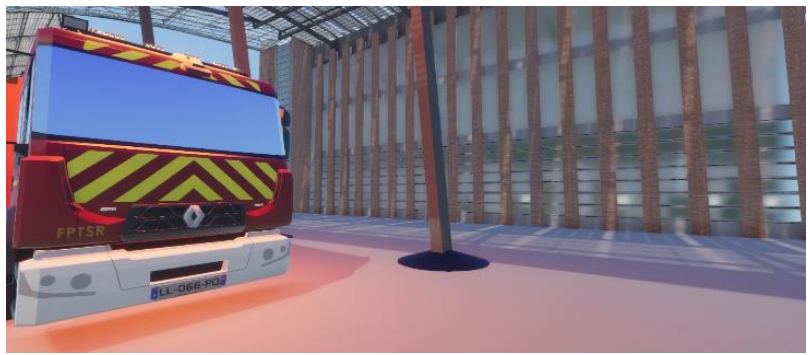

Figure 4: Firetruck near the building

The scenario ends when the firefighters who are better equipped for the situation approach (Fig 4).

\section{ARChitecture}

The design of this simulation led to a more in-depth project. For this use case, the environment is a digital replica of our lab. It has been programmed under UnityC 2019.3.2f1 with the default settings to integrate the Asset SteamVR $\odot$ and Photon Unity Engine 2@. SteamVR@ makes it possible to quickly have a VR situation in our 3D environment and finally have little interaction to develop for the VR. The simulation uses the High Rendering Pipeline technology of the Unity ${ }^{C}$ graphics engine which is a very recent technology that gives a more advanced graphics rendering.

To enhance user collaboration, the simulation includes a major new element compared to the previous use case: voice communication. Depending on their distance, the players will be able to talk to each other to hear each other and exchange information.
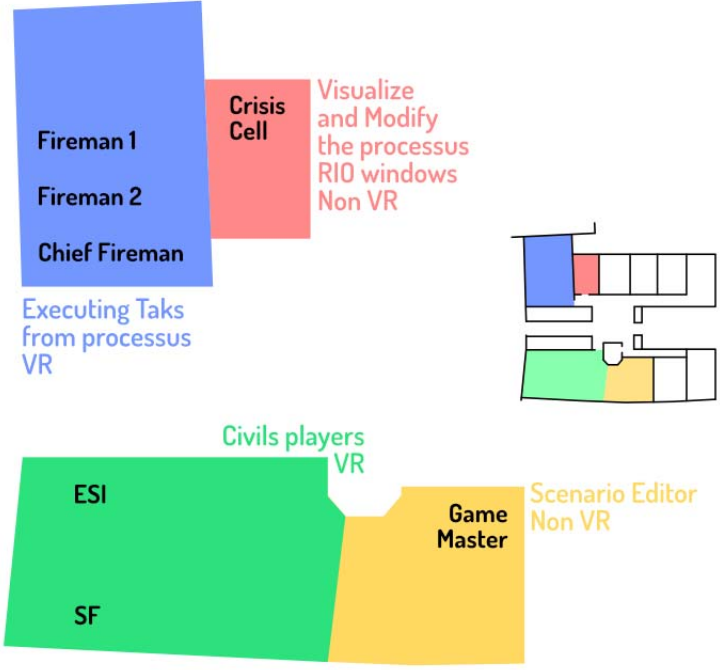

Figure 5: Deploy Plan 
The most challenging and the most interesting part of this demo is the architecture between the different services (Fig 6). Indeed, assembling the non-playable characters with the immersion players while having created a dynamic environment. The latter evolves according to an external person called Game Master who writes the scenario in real-time from a computer or a Windows tablet. The demo of the previous Use Case did not allow for the evolution of the process deduced at launch. Here the scenario can evolve and the actions that the players will carry out there is a renewal of the terms in the process. This will influence a different deduction.

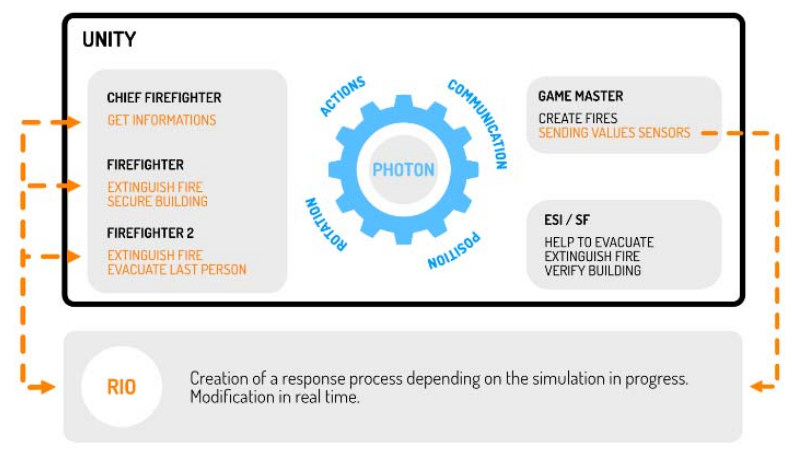

Figure 6: Architecture System

Compared to our previous simulation where the players had their tools directly embedded (e.g. the firefighter carrying an extinguisher), here the tools are integrated into the environment and this involves the players more in their roles. They must follow the process to not make mistakes in the virtual world. For example, fire extinguishers are placed in the building but have been used by the civilians, so they will have to use those near their emergency vehicle and therefore take them from the start to avoid going back and forth. The addition of different elements integrates more complexity during this immersion and can more easily modulate the scenario editor.

\subsection{Scenario Editor}

We have therefore set up a new central dynamic that we call Game Master, this person oversees writing the scenario in real-time and has the power to react according to the actions of the players.

For a question of accessibility, this new player has been designed to carry out these tasks outside of a Virtual Reality immersion. He is therefore located in front of a screen from a tablet or a computer with a top view of the place and can see each person in the environment and declare fires. The latter can be set and triggered in most of the school's rooms. The fire will spread in each room as the simulation unfolds. But the editing can be made in immersion with a lot of interactions.

When the player clicks or touch on one of the locations in the environment, a meeting room for example. An interface is displayed indicating the possibility to create a fire. The parameters are quite simple:

- type of fire

- $\quad$ size of the source object

- $\quad$ when to start the fire (with a delay or not)

Depending on what is started the fire behaves almost naturally by a limited propagation system to be easily adjusted. Several sensors located in the building are connected to our decision support platform and will be able to send information according to the situation and the places where smoke is present.

The Game master can use tactile support or not but it is possible to continue this tactile extension by providing support such as a tablet or a phone that could use Augmented Reality but also in exploring an immerged view. These points are exactly what we are going to work on next. This will lead to a more accessible immersion while being more agile.

\subsection{Virtual environment}

In addition to being able to see teammates, the addition of voice communication functionality is also an important point as it allows players to talk to each other without going through a system external to the simulation. During the game, the players will be able to choose different channels to have a parallel with an equivalent of a radio where the firefighters can communicate without any distance problem.

Specifically, we have 1 Game Master, 3 firefighters and 2 civilians. Each with a different course with appropriate tasks. The different objectives can change depending on the situation. This effect makes the scenario more dynamic.

Players will be surrounded by a host of non-player characters with civilian roles modelled and animated to fit into the set. They will have several animations including phone calls, typing or chatting. They are integrated to amplify the immersion of the players. The look is somewhere between a low-polished style and a realistic style. The characters are easily animated on a generic basis while keeping a modular design.

One of the difficulties of this use case has been to reproduce an environment like the one that exists. It took us several months to recreate the environment so that it could be used in several simulations. Using 2D plans but also 3D scans with drones, the school could be reconstructed to form a building close to the existing one. In this one, the model is simplified to guarantee stability in terms of performance.

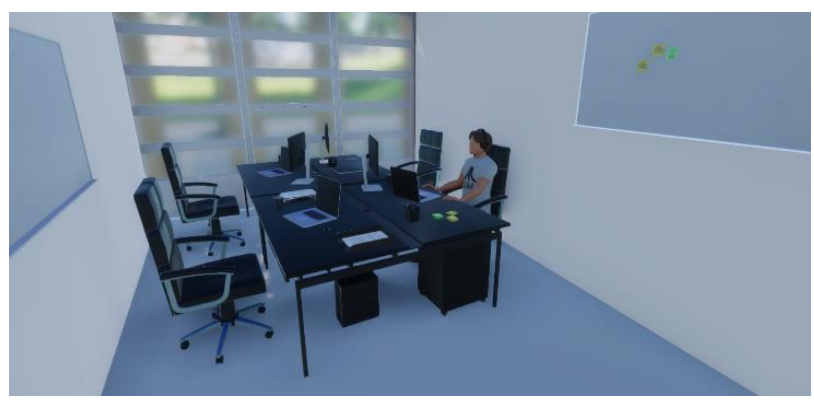

Figure 7: Office with one non-player character

The various tools and other useful objects are anchored in the environment and can be unhooked by the player for use. For example, an extinguisher will be at the same place that in the reallocation. The place of the simulation therefore includes dynamic objects programmed to be used or not by the players. It is therefore up to them to find these elements and use them. 
With increased agility, the simulation offers a multitude of interventions. The environment is directly integrated into an existing place. It allows having real time-sensitive sensors present on the real site. This setting is amplified by the presence of characters with realistic behaviour. And having made the tools useful in their dedicated spaces and not fixed to their hands from the start adds a significant difficulty and coherence.

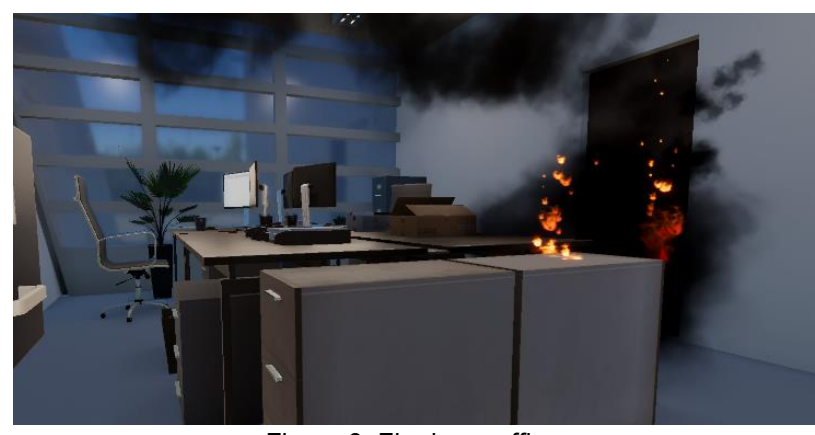

Figure 8: Fire in an office

Here you can see an example of an office with a fire in it (Fig 8). The smoke is close to the ceiling and will be more and more intense if the fire is not extinguished.

\subsection{Decision support tool}

This simulation needed to provide a certain amount of data to be able to deduce a coherent process when orchestrating the tasks to be carried out. Several smoke sensors are present in the establishment and we have reproduced them in Unity $\subset$ as well as on the decision support platform R-IO Suite [3]. They are connected and will be able to exchange information depending on the situation. Each sensor has an alert threshold on Unity $\subset$ when fire and smoke are close to a sensor with a certain intensity it sends to the decision support platform the information that the sensor detects smoke.

The decision support platform will send each player a set of tasks to perform. The purpose here is not to explain their handling but rather to indicate the directions as well as the fruitfulness of the collaboration between the different units. Thanks to a tablet attached to their arm, they can see the tasks they have received. Their tasks may have a description with coordinates but also details of the dangerousness. For example: Securing the building will have a description: Evacuate the gas bottle in office 0A02. Once completed, each player will be able to update the process by clicking on Send Update on their tablet (Fig 9).

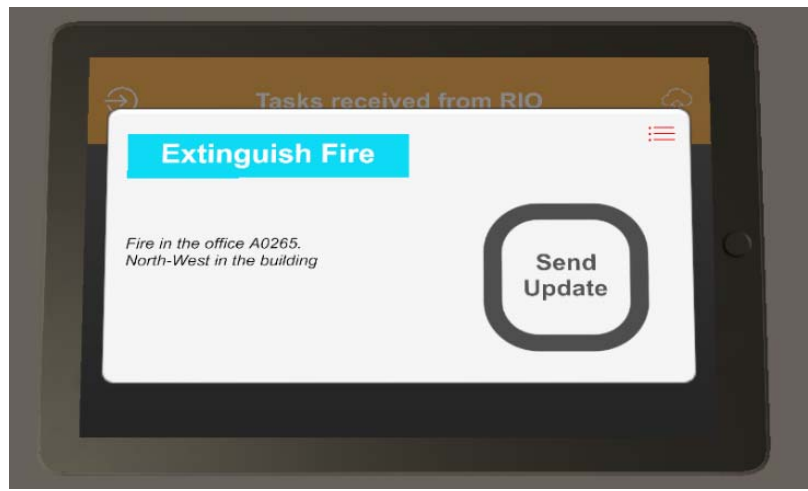

Figure 9: Tablet connected to the decision support

To install the simulation, all you need to do is to connect each pc by making public information such as their IP address. This will allow our decision support tool to exchange with each player individually. They will communicate with basic web requests.

The connection with our decision support platform is more dynamic than in the previous use case, which was fixed from start to finish. This scenario performs two different processes in a short period with a significant change in tasks and their priorities.

Depending on the situation, the sensors will modify the process. And this will modify the tasks to be performed by the players by adding more information if possible.

\section{OUTCOMES}

\subsection{Results}

The result is the sum of the work carried out by the feedback from the experts who witnessed our experiences but also from the users who were able to share their feelings and indicate valuable corrections. To find out if our solution works, we have carried out a battery of tests, some of which were filmed. The aim is to see how well the application offers a wide range of scenarios. The anchored points will establish a structure for our results. People have freedom outside these points that are relevant to observe. With several tests, we have been able to test the efficiency of the simulation. It is interesting to observe the different dynamic factors, which offer the necessary modularity to obtain an agile scenario.

A capture was carried out to effectively show our results. It can be found as "Unique immersion experience inside the IOMEGA VR platform" on the YouTube platform, here at https://youtu.be/FdO TSOA40. This video takes place in one single shot and focuses on the different essential elements of our simulation.

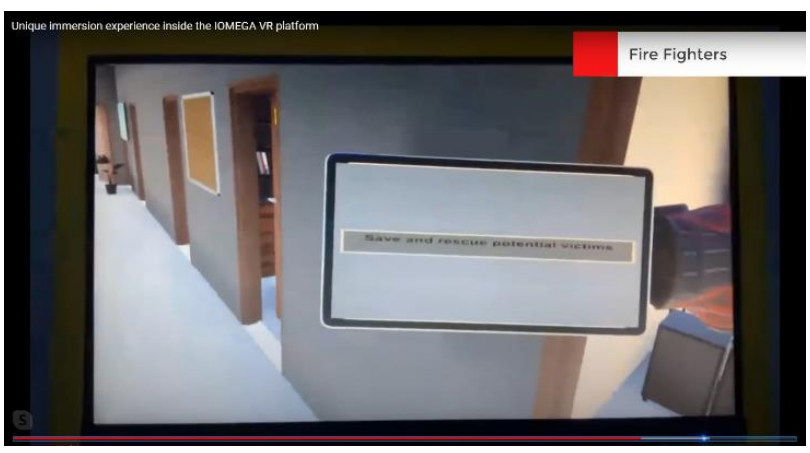

Figure 10: Image from the YouTube video

\subsection{Evaluation}

Through this development, we have been able to discover difficulties, particularly through having people who rarely use Virtual Reality and therefore had to become familiar with the controllers. The HTC Vive $C$ controllers are not known for their ergonomics and the user experience can be very difficult when the person is an amateur, especially if he or she is not a video game player. The majority of users admit that they have never had experiences using virtual reality and have been disturbed by their integration in an environment they are familiar with. They have been able to learn and navigate in their environment and master interactive objects. All this to fulfil their roles.

Simulation is highly dependent on manipulations in the virtual world. If one of the VR players makes a mistake, forgets, misunderstands, or interacts badly with an element it can completely disrupt the simulation. Beyond the user experience, there are still hardware and development problems. It can happen 
that a loss of a tracking break or a fire does not synchronize in multiplayer for all users. These points need to be more robust in the future. As our HTC Vive Pro $\odot$ devices are wireless, we have had several difficulties with tracking that could interrupt. It is possible that for the next use case we will test on two methods a wired and a wireless one to confirm this bias.

The evolutionary presence of the non-player characters fulfils its role perfectly. Despite a rather limited number of animations, their fidelity and their settings improve the feeling of realism for most of the immersed players. During the simulation, the players could notice the absence of sound from the unplayed civilians. It is planned to enhance discussions or shouting to better integrate the non-player characters and their interactions in the environment.

With this simulation, it is easy to reproduce a situation and to observe it. Each test can have a different course from other thanks to the modularity that can be set up. Nevertheless, we have fixed the elements described in the scenario.

Referring to figure 1, the expectations regarding collaboration and interactions correspond with the results. We can see that the business processes are well respected and well replicated.

Knowledge: It is not an in-depth direction that is set on this simulation.

Action: The development is still too recent to allow a realistic approach to interactions and actions with tools in the field. For the moment, the symbolic approach of a fire extinguisher is sufficient. The interaction with objects in the environment is more subtle because it is coherent. However, it requires knowledge of the joystick keys to carry out the interactions easily. Once this learning period is over, the gesture is carried out naturally. The aim is not to reproduce gesture by gesture the right movements to use a particular fire extinguisher. The interactions are simplified but remain unexplained which can be frustrating at the beginning. Once understood this is functional and it accentuates the ergonomics of the user experience.

Process: Although the exercise did not have this aim, the experts applied their protocols and positioned themselves in the same situations as in reality. Putting one's hand close to a suspicious door is a consistent technique. Placing a hand on a door to check the temperature of the room for example. This reinforces the effectiveness of the management of our research.

Collaboration: They will coordinate well according to their trade assignment. Important information is exchanged with the voice channels. And their collaboration is more refined thanks to vocal interaction.

\section{CONCLUSION}

This experiment was complex to develop because it was intended to be close to reality. It is necessary to consider a plurality of parameters that have been suggested by professionals such as the spread of fire and the appearance of smoke. The requested requirement made it possible to carry out this simulation and to satisfy the participants as well as the professionals.

Increasing the potential of the Game Master in terms of interaction on the simulation but also in terms of visualization is one of the avenues that will have to be advanced. It is easy to continue tactile $2 \mathrm{D}$ support as here but it is envisaged to imagine its role in virtual immersion or to have exploitation using augmented reality.

Integrating a system where each player could have his summary of his simulation to observe his course and potentially his mistakes. A dashboard summarising the actions of the game would be saved. The point could be, for example, to emphasize a civil loss due to a loss of time or a bad method follow up. This could be exploited by the Game Master who would have access to all his interventions.

The success of this experiment has confirmed the questioning around the subject and adds new and very interesting issues. It is possible to replicate situations to derive results concerning the behaviour and actions of the players. This creates a safe context where nothing dangerous is involved and everything is virtual. The application can be used in multiplayer and can be played all over the world.

Future simulations will include new interactions, not just creating fires and a parallel of different situations that the Game Master will have to manage with further interaction. Several avenues have already been discussed with partner companies.

The scenarios could have consequences for every other place. The vision may have several scenario managements states. The vision of the scenario writer will not be there to see the whole site or to generate a problem in a particular room.

The aim will be to have several sites, each with its particularities. It is planned to have an industrial site categorised as CBRN, another historical site.

In future cases, it will be necessary to integrate the opportunity to join as a civilian in addition to the professional trades to establish a civilian opportunity during the simulation. Dealing with Virtual Reality provides real support to immerse different people. This technology offers an answer that positions itself as a solution.

\section{References}

[1] L. Ardila, I. Pérez-Llopis, C. E. Palau, and M. Esteve, 'LVC Training Environment for Strategic and Tactical Emergency Operations', in Proceedings of the Tenth International Conference on Information System for Crisis Response and Management, Baden-Baden, Germany, 2013.

[2] L. Bacon, L. MacKinnon, A. Cesta, and G. Cortellessa, 'Developing a smart environment for crisis management training', $J$ Ambient Intell Human Comput, vol. 4, no. 5, pp. 581-590, Oct. 2013.

[3] F. Benaben et al., 'A conceptual framework and a suite of tools to support crisis management', in HICSS 2017-50th Hawaii International Conference on System Sciences, 2017, p. 237.

[4] A. Conges, F. Benaben, O. Pierre, F. Savic, O. Chabiron, and M. Lauras, 'On the usage of Virtual Reality for Crisis Management exercises in Critical Industrial Sites', in Proceedings of the Sixteenth International Conference on Information System for Crisis Response and Management, Valencia, Spain, 2019.

[5] A. Conges, A. Evain, F. Benaben, O. Chabiron, and S. Rebiere, 'Crisis Management Exercises in Virtual Reality', presented at the 2020 IEEE Conference on Virtual Reality and 3D User Interfaces, Atlanta, GA, USA, USA, 2020, pp. 87-92. [6] L. C. Dukes and L. F. Hodges, 'Development of a scenario builder tool for scaffolded virtual patients', in 2014 IEEE Virtual Reality (VR), 2014, pp. 131-132.

[7] J. Falah et al., 'Virtual Reality medical training system for anatomy education', in 2014 Science and Information Conference, 2014, pp. 752-758.

[8] P. Gallerati, M. Maisano, C. Valiante, A. Conte, and D. Casciaro, 'The Use of Virtual Reality and Simulators for Emergency Management Training Increases Employees’ Role Awareness, Commitment and Information Retention, While Helping Companies to Stay Compliant.', in Offshore Mediterranean Conference, 2019.

[9] F.-M. Gerard, 'Savoir... oui mais encore ?', p. 13, 2000.

[10] W. L. Heinrichs, P. Youngblood, P. M. Harter, and P. Dev, 'Simulation for Team Training and Assessment: Case Studies of 
Online Training with Virtual Worlds', World J Surg, vol. 32, no. 2, pp. 161-170, Feb. 2008.

[11] P.-A. Heng et al., 'A Virtual-Reality Training System for Knee Arthroscopic Surgery', IEEE Trans. Inform. Technol. Biomed., vol. 8, no. 2, pp. 217-227, Jun. 2004.

[12] J. Holopainen, A. Lähtevänoja, O. Mattila, I. Södervik, E. Pöyry, and P. Parvinen, 'Exploring the Learning Outcomes with Various Technologies - Proposing Design Principles for Virtual Reality Learning Environments', in HICSS 2020-53rd Hawaii International Conference on System Sciences, 2020.

[13] S. G. Izard, J. A. Juanes, F. J. García Peñalvo, J. M. G. Estella, M. J. S. Ledesma, and P. Ruisoto, 'Virtual Reality as an Educational and Training Tool for Medicine', J Med Syst, vol. 42, no. 3, p. 50, Feb. 2018.

[14] S. Kang, J. Chanenson, P. Ghate, P. Cowal, M. Weaver, and D. M. Krum, 'Advancing Ethical Decision Making in Virtual Reality', in 2019 IEEE Conference on Virtual Reality and 3D User Interfaces (VR), 2019, pp. 1008-1009.

[15] P. K. Kwok, B. K. P. Chan, and H. Y. K. Lau, 'A Virtual Collaborative Simulation-based Training System', in Proceedings of the 10th International Conference on Computer Modeling and Simulation, New York, NY, USA, 2018, pp. 258-264.

[16] M. Kyan et al., 'An Approach to Ballet Dance Training through MS Kinect and Visualization in a CAVE Virtual Reality Environment', ACM Trans. Intell. Syst. Technol., vol. 6, no. 2, pp. 1-37, May 2015.

[17] F. Lateef, 'Simulation-based learning: Just like the real thing', $J$ Emerg Trauma Shock, vol. 3, no. 4, pp. 348-352, 2010.

[18] J. Lester, 'Pathfinder Linden's guide to getting started in Second Life', in Proceedings of the Second Life Education Workshop at the Second Life Community Convention, San Francisco, 2006, vol. 26.

[19] K. Meesters and Y. Wang, 'Information Management in Largescale Disaster Exercises: An Integrated Perspective', in HICSS 2020-53th Hawaii International Conference on System Sciences, Hawaii, 2020, p. 10.

[20] T. A. Mikropoulos and A. Natsis, 'Educational virtual environments: A ten-year review of empirical research (19992009)', Computers \& Education, vol. 56, no. 3, pp. 769-780, Apr. 2011.

[21] G. S. Ruthenbeck and K. J. Reynolds, 'Virtual reality for medical training: the state-of-the-art', Journal of Simulation, vol. 9, no. 1, pp. 16-26, Feb. 2015.

[22] J. Saunders, S. Davey, P. S. Bayerl, and P. Lohrmann, 'Validating Virtual Reality as an Effective Training Medium in the Security Domain', in 2019 IEEE Conference on Virtual Reality and $3 D$ User Interfaces (VR), 2019, pp. 1908-1911.

[23] N. E. Seymour et al., 'Virtual Reality Training Improves Operating Room Performance', Ann Surg, vol. 236, no. 4, pp. 458-464, Oct. 2002.

[24] P. Stefan et al., 'An AR edutainment system supporting bone anatomy learning', in 2014 IEEE Virtual Reality (VR), 2014, pp. $113-114$.

[25] E. J. Wood, 'Problem-Based Learning: Exploiting Knowledge of how People Learn to Promote Effective Learning', Bioscience Education, vol. 3, no. 1, pp. 1-12, May 2004.

[26] 'Learning | Definition of Learning by Oxford Dictionary on Lexico.com also meaning of Learning', Lexico Dictionaries | English. [Online]. Available: https://www.lexico.com/definition/learning. [Accessed: 02-Oct2020]. 